\title{
Ruptured Intrarenal Arterial Aneurysm in a Patient With Granulomatosis With Polyangiitis
}

Johanna Schneider, MD, Department of Nephrology, University Medical Center, Faculty of Medicine, University of Freiburg; Nils Venhoff, MD, Department of Rheumatology and Clinical Immunology, University Medical Center, Faculty of Medicine, University of Freiburg; Gerd Walz, MD, Department of Nephrology, University Medical Center, Faculty of Medicine, University of Freiburg, Freiburg, Germany. Address correspondence to Dr. J. Schneider, University Medical Center, Faculty of Medicine, University of Freiburg, Hugstetter Straße 55, D-79106 Freiburg, Germany. Email: johanna.schneider@uniklinik-freiburg.de. Written consent for this case report was obtained from the patient reported. The Ethics Committee of the Albert-Ludwigs University of Freiburg did not have any ethical or legal objections to the publication of this case report.

We present a rare case of antineutrophil cytoplasmic antibodies (ANCA)-associated small vessel vasculitis complicated by recurrent bleeding from intrarenal aneurysms, which is typically associated with polyarteritis nodosa, a form of vasculitis affecting medium-sized vessels.

The patient, a 58-year-old man, presented with fever, arthralgia, and acute kidney injury associated with hematuria and proteinuria. Urine sediment analysis revealed acanthocytes and erythrocyte casts; C-reactive protein was highly elevated and anti-proteinase 3 (PR3)-ANCA were detectable. Computed tomography scan showed a cavity of the left upper lung (Figure 1A). Histologic examination of a lung biopsy revealed granulomatous inflammation with necrotizing small vessel vasculitis. Granulomatosis with polyangiitis was diagnosed, affecting both the lung and kidneys. The patient rapidly developed acute respiratory failure with pulmonary hemorrhage and left-side abdominal pain associated with kidney failure, requiring dialysis. Arterial angiography revealed multiple aneurysms of intrarenal arteries, including a ruptured aneurysm (Figure 1B). Bleeding from the intrarenal aneurysm was stopped by coiling the affected artery. The patient was treated intravenously with high-dose glucocorticoids (GC) combined with cyclophosphamide (CYC) for 6 days (1.8 g total). Because of the potentially life-threatening disease, treatment was escalated with plasmapheresis followed by rituximab $(2 \times 1 \mathrm{~g})$. The patient showed adequate treatment response but developed a major relapse only 7 weeks later with multiple lacunar ischemic strokes and acute bleeding of the contralateral kidney. The bleeding stopped spontaneously, and additional intravenous GC with CYC pulse therapy resulted in clinical improvement with normalization of the anti-PR3-ANCA. The lung granuloma decreased in size (Figure 1C); however, kidney function did not recover.
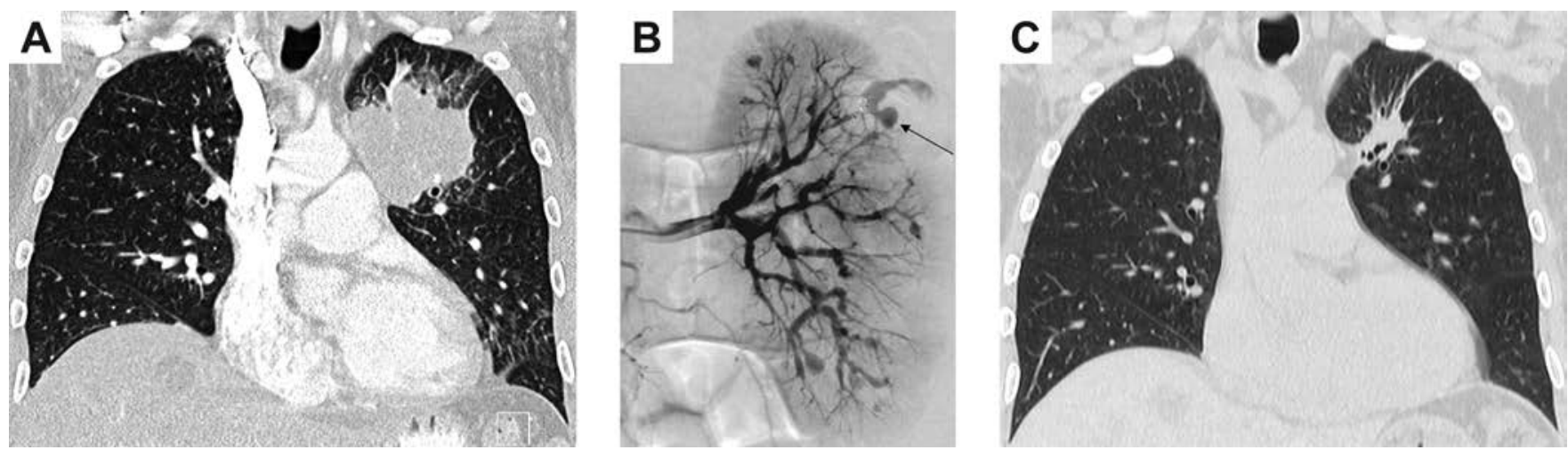

Figure 1. (A) CT scan showed a cavity of the left upper lung. (B) Arterial angiography revealed multiple aneurysms of intrarenal arteries, including a ruptured aneurysm. (C) Decrease in size of the lung granuloma. CT: computed tomography. 